\title{
Tracheostomy During the COV-SARS-CoV-2 pandemic: Recommendations from the New York Head and Neck Society
}

\author{
Brett A. Miles, DDS, MD ${ }^{1}$ \\ ${ }^{1}$ Mount Sinai, Icahn School of Medicine
}

April 28, 2020

Brett A. Miles DDS MD ${ }^{1}$, Bradley Schiff $\mathrm{MD}^{2}$, Ian Ganly MD MS PhD ${ }^{3}$, Thomas Ow MD MS ${ }^{2}$, Erik Cohen $\mathrm{MD}^{5}$, Eric Genden MD MPH${ }^{1}$, Bruce Culliney MD ${ }^{1}$, Bhoomi Mehrotra $\mathrm{MD}^{6}$, Steven Savona $\mathrm{MD}^{6}$, Richard J. Wong $\mathrm{MD}^{3}$, Missak Haigentz $\mathrm{MD}^{5}$, Salvatore Caruana $\mathrm{MD}^{7}$, Babak Givi MD ${ }^{8}$, Kepal Patel MD ${ }^{8}$, Kenneth $\mathrm{Hu} \mathrm{MD}^{8}$

${ }^{1}$ Icahn School of Medicine at Mount Sinai, New York, NY

${ }^{2}$ Montefiore Medical Center, Albert Einstein College of Medicine, Bronx, NY

${ }^{3}$ Memorial Sloan Kettering Cancer Center, Weill Cornell Medical College, New York,

${ }^{4}$ Cancer Institute at St. Francis Hospital, New York, NY

${ }^{5}$ Morristown Medical Center, Leonard B. Kahn Head and Neck Cancer Institute, Morristown, NJ

${ }^{6}$ Northwell Cancer Institute, Monter Cancer Center, Lake Success, NY

${ }^{7}$ Columbia University, New York, NY

${ }^{8}$ NYU Langone Health, New York, NY

\section{Abstract: Background}

The rapid spread of SARS-CoV-2 in 2019 and 2020 has resulted in a worldwide pandemic.[1-4]The dramatic proinflammatory effects of COV-SARS-CoV-2 results in a wide variety of clinical presentations, however severe pulmonary inflammation, effusions, and rapid respiratory compromise are a hallmark of this disease.[57]Subsequent pneumonia, acute respiratory distress syndrome and death have been reported not infrequently. The result of this pandemic is a large and increasing number of patients requiring endotracheal intubation and prolonged ventilator support.[8-13] Certainly, the rapid rise in endotracheal intubations coupled with prolonged ventilation requirements will lead to an increase in tracheostomy procedures in the coming weeks and months. $[14,15]$

While generally a well-tolerated and safe procedure, the risks and benefits of tracheostomy in terms of outcomes, pulmonary care, and risks to the health care team remain unknown [16, 17]. Fortunately, while not perfect, rapid testing protocols have allowed us the ability to detect active infection in patients who are affected by SARS-CoV-2.[18-21] What is clear is that the upper aerodigestive tract, the nasopharynx and the trachea harbor a high viral load during the acute stages of the infection.[22-24] Therefore, performing tracheostomy in the setting of active COV-SARS-CoV-2 when necessary, poses a unique situation, with unique risks and benefits for both the patient and the health care providers. The risk of this procedure has to be balanced with the known risks of prolonged intubation, primarily tracheal and subglottic stenosis the management of which can be problematic if significant mucosal injury and subsequent stenosis occurs. 
The New York Head and Neck Society is a non-profit organization founded in 1979, which encourages the exchange and advancement of scientific knowledge relative to the management of head and neck cancer and includes several member institutions including Columbia University Medical Center, Cornell Medical College, Icahn School of Medicine at Mount Sinai, Memorial Sloan Kettering Cancer Center, New York University Medical Center, Montefiore Medical Center Albert Einstein College of Medicine, and has several other affiliate institutions in the greater New York City area. The New York Head and Neck Society has collaborated on this document to provide guidance on the performance of tracheostomy during the SARS-CoV-2 pandemic.

\section{Recommendations:}

\section{Monitor endotracheal and tracheostomy tube cuff pressures Q4 hours.}

In patients who are intubated, especially in prolonged intubations $>72$ hours the risk of tracheal stenosis increases over time. Teams managing these patients should stress that all intubated patients have Q4 hour cuff pressure check with goal of approximately $30 \mathrm{~mm}$ $\mathrm{Hg}$ if feasible given the vent parameter requirements, as pressures higher than 30mmg Hg may result in pressure necrosis. Certainly, adequate pressure to avoid cuff leakage and aerosolization is critical when managing SARS-CoV-2 patients, but it should be recognized that unnecessarily high cuff pressures are also problematic. The minimum cuff pressure required to create an adequate seal should be individualized for each patient and verified frequently by care providers. This is a dynamic process and frequent adjustments may be indicated depending on ventilation parameters. Prevention of tracheal mucosal pressure necrosis, resulting tracheal and cricoid chondritis, and subsequent stenosis is critical in the SARS-CoV-2 population.[25] [26]

SARS-CoV-2 testing via RT-PCE detection platform for SARS-CoV-2 and pansarbecovirus detection is recommended for all patients who are under consideration for tracheostomy. Keeping in mind that that data surrounding accuracy of the test during the pandemic is forthcoming, and false negatives are a real possibility.[27] The test may be performed a second time if clinical suspicions or institutional policy warrants repeat testing prior to high risk procedures.

\section{Delay timing of tracheostomy until 21 days post intubation if feasible.}

When determining the appropriate time of tracheostomy in the SARS-CoV-2 patient a variety of factors are considered, and certainly individual cases may have mitigating circumstances which lead to the decision to perform tracheostomy. However, for the majority of patients, health care teams should seek to capitalize on the intersection of the risk of contamination/infection and decreasing viral load in the upper and lower airway over time with the risks of prolonged intubation(i.e. tracheal stenosis). While the overall risk of tracheal stenosis secondary to prolonged intubation depends on a variety of factors, reported rates of severe, symptomatic stenosis are generally in the 1-2\% range when modern low-pressure cuffs are utilized.[28-32] Therefore in light of the relatively low risk of clinically relevant stenosis, and despite the traditional 10-day cutoff for increased stenosis risk used by many practitioners in the general population, when dealing with a SARS-CoV-2 patient the risk for symptomatic or severe tracheal stenosis is acceptable in light of the significant risks of tracheostomy in the acute phase of the infection during periods of highest viral loads. The decreasing viral load, while logarithmic in nature is somewhat variable and high viral loads have been observed somewhat late in the course of the infection in critically ill patients.[23, 24, 33](Figure 1,2) Therefore when feasible, waiting until approximately 21 days after intubation is recommended prior to consideration of tracheostomy for the majority of cases in order to avoid exposing health care teams to increased risk. Certainly, earlier tracheostomy may be medically indicated in some situations depending on the clinical situation and we recognize the potential need to perform tracheostomy more urgently. Tracheostomy should not be delayed regardless of SARS-CoV-2 status in life saving situations, 
or in situations where the tracheostomy would significantly improve the prognosis of the patient. Alternative emerging strategies in the management of SARS-CoV-2 critically ill patients, such as extracorporeal membrane oxygenation, antiviral therapy, and convalescent plasma therapy may also be considered by the team but the available data and decision making regarding this is beyond the scope of these recommendations. [34-38] Clearly these are multidisciplinary decisions which will be individualized depending on the patient and institutional expertise. In addition, it should be noted that avoiding tracheostomy in high mortality risk patients is critical. If the primary team managing the patient determines that there is an extremely high risk of mortality in the near future, or that the patient has a high likelihood of withdrawal of care, the risks of tracheostomy should be avoided in this situation. Patients, with significant medical comorbidities, ARDS/severe respiratory failure, and a low chance of recovery who are infected with SARS-CoV2 , should be carefully evaluated, and discussions with family members, consultants, institutional ethics committees, and the treating team should focus on overall prognosis and goals of care, prior to performing tracheostomy as a routine matter of care. These decisions are highly individualized and rely on solid communication amongst team members managing these high-risk patients.

\section{Tracheostomy Technical Considerations and Recommendations.}

While the exact technical details regarding tracheostomy will depend on the situation and procedural protocols and technical expertise, there are some specific technical aspects related to the SARS-CoV-2 (and other viral pandemics) which should be considered. Ideally the procedure should be performed at bedside in the ICU in a negative pressure room or using a portable HEPA filtration system to avoid patient transportation and contamination of other areas in the medical center. If it is necessary to perform the procedure in the OR, a specific OR cluster should be designated to avoid contamination of additional OR resources for non- infected patients.

In addition to standard airborne and droplet precautions, techniques to minimize aerosolization of the virus during the procedure include the following: paralysis to prevent coughing, consider glycopyrrolate to reduce secretions, preoxygenation and cessation of ventilation during the tracheostomy procedure, utilization of closed suctioning systems, avoiding monopolar electrocautery and using cold instrumentation when feasible, minimizing suctioning and bronchoscopy during the procedure, and ensuring the cuff is inflated prior to resuming ventilation so the circuit is closed. In addition to standard open tracheostomy, percutaneous/dilational tracheostomy techniques have been evaluated extensively in the literature and have been shown to be a safe alternative to traditional open surgical tracheostomy.[39-41] Understandably, the techniques utilized when performing tracheostomy will vary based on patient characteristics, provider expertise, and institutional experience. While data is limited, techniques which avoid opening the airway and are closed such as a percutaneous dilational technique, may be preferential in the setting of active SARS-CoV-2 infection. $[42,43]$ Therefore if there are no anatomical or other contraindications, percutaneous dilational tracheostomy may be considered if the expertise is available. It should be kept in mind that the decrease in aerosolization during percutaneous tracheostomy only holds true if airway manipulation (i.e. bronchoscopy) is not performed, and while there have been some associated higher complication rates with blind percutaneous tracheostomy compared to bronchoscopic technique, ultrasound guided techniques have been shown to be non-inferior to bronchoscopic techniques.[44-46]. Therefore, if considering a percutaneous tracheostomy, a closed ultrasound guided technique is recommended for SARS-CoV-2 patients.

\section{Use of appropriate PPE during tracheostomy procedures for active SARS-CoV-2.}

While there is limited data on the current pandemic to fully inform current recommendations, certainly performing tracheostomy in an actively infected SARS-CoV-2 patient is a high-risk procedure for health care workers. [47] Health care personnel performing the tracheostomy should wear at minimum: 
Waterproof cap, goggles with an anti-mist screen, N95 mask, impermeable operating room surgeon's gown and gloves, and a transparent plastic facial shield worn outside the goggles and N95 effective to filter 99.5\% particles larger than $0.75 \mu \mathrm{m}$. [48] The minimum number of health care workers required to perform the procedure should be present to prevent unnecessary exposures. The effectiveness of the N95 mask in the prevention of SARS-CoV-2 infection during tracheostomy procedures remains unknown, but given the high risk consideration for power air purifying respirator (PAPR) systems for personnel performing tracheostomy should be entertained, and these systems should be used when available in situations of active infection, or suspicion of high viral loads, as there is some evidence of superior protection (PAPR provides 2.5 to 100 times greater protection than the N95, when staff are appropriate trained.[47, 49, 50] Certainly the effectiveness of N95 and PAPR in this situation has not been compared in a head to head trial, and therefore the use of PAPR vs. N95 will depend on institutional resources and policy, and the clinical situation.

\section{Avoid emergent tracheostomy if possible.}

Techniques to manage the acute airway with endotracheal intubation, video laryngoscope and fiberoptic intubation should be utilized if possible to avoid emergent tracheostomy in SARS-CoV-2 patients due to the high risk of unsafe conditions and health care worker contaminations.[48] Similarly, intubation techniques (i.e. rapid sequence intubation) which avoid mask ventilation, prolonged open airway manipulation are recommended when appropriate. When life threatening airway obstruction occurs in a setting in which intubation is not possible, healthcare workers should perform the tracheostomy with the above noted PPE keeping in mind that PAPR respirator use is often not feasible or available in emergent situations. In situations where CPR is being performed, chest compressions should be held at the time the airway is entered, until the airway is secured and the cuff inflated on the device, to minimize health care worker exposure.

\section{Appropriate post tracheostomy management.}

The post-tracheostomy management should also be mentioned, as in addition to routine tracheostomy care, there are some considerations for SARS-CoV-2 patient. Securing circuits properly and avoiding unnecessary humidification systems may reduce the risk of unexpected circuit disconnection and aerosolization leading to exposure. The circuit should remain closed as much as possible, and closed line suctioning should be used. Heat moister exchangers with viral filters and HEPA filtration should be used when possible. Tracheostomy tube changes should be avoided, and only performed in cases of cuff failure, or emergent situations.

\section{Organize an appropriate team.}

While the members of the health care team performing tracheostomy vary across institutions, team members may include surgeons, medicine/intensivists, anesthesiologists, respiratory therapists, nurses, and other ancillary staff required during these procedures. The importance of appropriate PPE/PAPR training and usage cannot be overstated in the setting of active SARS-CoV-2 infection. Teams who perform the procedure regularly will be more efficient and less likely to be unfamiliar with the procedure or appropriate health care protective measures and infection control. The inclusion of trainees such as residents and fellows during these procedures requires careful consideration and will vary based on institutional policies. Currently there is limited data on the host innate immune status of SARS-CoV-2 infected patients.[51] Consideration of the inclusion of health care workers who have previously been exposed and subsequently recovered from documented SARS-CoV-2 infection may be warranted. While the exact timing of immunity and subsequent safety for the return of health care providers infected with SARS-CoV-2 remains unknown, sufficient antibody responses have been documented to occur between days 15-20, or approximately two weeks after the onset of symptoms.(Figure 3)[23] Inclusion of these individuals on these teams may allow for high risk procedures to be performed by health care workers who have mounted an immune response to the virus, depending on institutional quarantine policies. Similarly, these individuals should not be involved in tracheostomy procedures or other airway procedures in non-infected patients due to the risk of iatrogenic infection with SARS-CoV-2 due to limited available data about the risks.[52]

\section{Conclusion:}


Tracheostomy in the SARS-CoV-2 infected patient represents a unique situation, with a unique set of risks and implications. When compared to traditional tracheostomy procedures in the setting of prolonged ventilation, SARS-CoV-2 represents a unique entity in terms of timing, indications, and infection control considerations which must be kept in mind when performing these procedures and managing patient's posttracheostomy. Additional resources are listed below.

\section{Summary Points:}

- Careful consideration of "who" and "when" when tracheostomy is planned.

- Careful consideration of the location and technique to avoid unnecessary risks to health care providers.

- When clinically appropriate, delay of tracheostomy procedures is recommended to allow for reduced viral load and decrease the risk of nosocomial infection to critical health care providers.

- Careful monitoring of EET cuff pressures to maintain appropriate seal to avoid aerosolization, while mitigating the risk of long-term tracheal complications.

- Appropriate PPE training and utilization, including N95 or PAPR when indicated is recommended for all patients undergoing tracheostomy, regardless of SARS-CoV-2 status, during the pandemic.

- Avoidance of unnecessary airway manipulation such as bronchoscopy, trach changes, with a focus on a closed-circuit ventilation, and utilizing ultrasound guidance for percutaneous tracheostomy.

\section{Resources:}

American Academy of Otolaryngology-Head and Neck Surgery

Tracheostomy Recommendations during COVID-19 Pandemic.

https://www.entnet.org/content/tracheotomy-recommendations-during-covid-19-pandemic

American Head and Neck Society Guidance for Tracheostomy during COVID-19 Pandemic.

https://www.ahns.info/wp-content/uploads/2020/03/Guidance-for-Surgical-Tracheostomy-and-Tracheostomy-

Tube-Change-during-the-COVID.pdf

Percutaneous Tracheostomy Technique:

https://www.hopkinsmedicine.org/tracheostomy/about/how.html

https://www.vumc.org/trauma-and-scc/sites/vumc.org.trauma-and-scc/files/public_files/Protocols/Tracheostomy\%202019.

Open Tracheostomy Technique:

https://www.mayoclinic.org/tests-procedures/tracheostomy/about/pac-20384673

https://my.clevelandclinic.org/health/treatments/17568-tracheostomy-care

https://medicine.uiowa.edu/iowaprotocols/tracheotomy-tracheostomy

\section{References}

1. Amodio E, Vitale F, Cimino L et al. Outbreak of Novel Coronavirus (SARS-Cov-2): First Evidences From International Scientific Literature and Pending Questions. Healthcare (Basel) 2020; 8.

2. Ashour HM, Elkhatib WF, Rahman MM, Elshabrawy HA. Insights into the Recent 2019 Novel Coronavirus (SARS-CoV-2) in Light of Past Human Coronavirus Outbreaks. Pathogens 2020; 9.

3. Dilcher M, Werno A, Jennings LC. SARS-CoV-2: a novel deadly virus in a globalised world. N Z Med J 2020; 133: 6-11. 
4. Hsu LY, Chia PY, Lim JF. The Novel Coronavirus (SARS-CoV-2) Epidemic. Ann Acad Med Singapore 2020; 49: 1-3.

5. Conti P, Ronconi G, Caraffa A et al. Induction of pro-inflammatory cytokines (IL-1 and IL-6) and lung inflammation by Coronavirus-19 (COVI-19 or SARS-CoV-2): anti-inflammatory strategies. J Biol Regul Homeost Agents 2020; 34.

6. Coronaviridae Study Group of the International Committee on Taxonomy of V. The species Severe acute respiratory syndrome-related coronavirus: classifying 2019-nCoV and naming it SARS-CoV-2. Nat Microbiol 2020; 5: 536-544.

7. Fu Y, Cheng Y, Wu Y. Understanding SARS-CoV-2-Mediated Inflammatory Responses: From Mechanisms to Potential Therapeutic Tools. Virol Sin 2020.

8. Giani M, Seminati D, Lucchini A et al. Exuberant Plasmocytosis in Bronchoalveolar Lavage Specimen of the First Patient Requiring Extracorporeal Membrane Oxygenation for SARS-CoV-2 in Europe. J Thorac Oncol 2020.

9. Kamel Boulos MN, Geraghty EM. Geographical tracking and mapping of coronavirus disease COVID19 /severe acute respiratory syndrome coronavirus 2 (SARS-CoV-2) epidemic and associated events around the world: how 21st century GIS technologies are supporting the global fight against outbreaks and epidemics. Int J Health Geogr 2020; 19: 8.

10. Lai CC, Liu YH, Wang CY et al. Asymptomatic carrier state, acute respiratory disease, and pneumonia due to severe acute respiratory syndrome coronavirus 2 (SARS-CoV-2): Facts and myths. J Microbiol Immunol Infect 2020.

11. Lai CC, Shih TP, Ko WC et al. Severe acute respiratory syndrome coronavirus 2 (SARS-CoV-2) and coronavirus disease-2019 (COVID-19): The epidemic and the challenges. Int J Antimicrob Agents 2020; 55: 105924 .

12. Li T. Diagnosis and clinical management of severe acute respiratory syndrome Coronavirus 2 (SARSCoV-2) infection: an operational recommendation of Peking Union Medical College Hospital (V2.0). Emerg Microbes Infect 2020; 9: 582-585.

13. Sun P, Qie S, Liu Z et al. Clinical characteristics of hospitalized patients with SARS-CoV-2 infection: A single arm meta-analysis. J Med Virol 2020.

14. Lombardi A, Bozzi G, Mangioni D et al. Duration of quarantine in hospitalized patients with severe acute respiratory syndrome coronavirus 2 (SARS-CoV-2) infection: a question needing an answer. J Hosp Infect 2020 .

15. Marraro GA, Spada C. Consideration of the respiratory support strategy of severe acute respiratory failure caused by SARS-CoV-2 infection in children. Zhongguo Dang Dai Er Ke Za Zhi 2020; 22: 183-194.

16. Niederman MS, Richeldi L, Chotirmall SH, Bai C. Rising to the Challenge of the Novel SARS-coronavirus2 (SARS-CoV-2): Advice for Pulmonary and Critical Care and an Agenda for Research. Am J Respir Crit Care Med 2020.

17. Ong SWX, Tan YK, Chia PY et al. Air, Surface Environmental, and Personal Protective Equipment Contamination by Severe Acute Respiratory Syndrome Coronavirus 2 (SARS-CoV-2) From a Symptomatic Patient. JAMA 2020.

18. Liu R, Han H, Liu F et al. Positive rate of RT-PCR detection of SARS-CoV-2 infection in 4880 cases from one hospital in Wuhan, China, from Jan to Feb 2020. Clin Chim Acta 2020; 505: 172-175.

19. Patel R, Babady E, Theel ES et al. Report from the American Society for Microbiology COVID-19 International Summit, 23 March 2020: Value of Diagnostic Testing for SARS-CoV-2/COVID-19. mBio 2020; 11. 
20. Pfefferle S, Reucher S, Norz D, Lutgehetmann M. Evaluation of a quantitative RT-PCR assay for the detection of the emerging coronavirus SARS-CoV-2 using a high throughput system. Euro Surveill 2020; 25.

21. Wang Y, Kang H, Liu X, Tong Z. Combination of RT-qPCR testing and clinical features for diagnosis of COVID-19 facilitates management of SARS-CoV-2 outbreak. J Med Virol 2020.

22. Pan Y, Zhang D, Yang P et al. Viral load of SARS-CoV-2 in clinical samples. Lancet Infect Dis 2020; 20: $411-412$.

23. To KK, Tsang OT, Leung WS et al. Temporal profiles of viral load in posterior oropharyngeal saliva samples and serum antibody responses during infection by SARS-CoV-2: an observational cohort study. Lancet Infect Dis 2020.

24. Zou L, Ruan F, Huang M et al. SARS-CoV-2 Viral Load in Upper Respiratory Specimens of Infected Patients. N Engl J Med 2020; 382: 1177-1179.

25. Kumar CM, Seet E, Van Zundert T. Measuring endotracheal tube intracuff pressure: no room for complacency. J Clin Monit Comput 2020.

26. Mehel DM, Ozdemir D, Celebi M et al. Classification of laryngeal injury in patients with prolonged intubation and to determine the factors that cause the injury. Am J Otolaryngol 2020; 102432.

27. Li D, Wang D, Dong J et al. False-Negative Results of Real-Time Reverse-Transcriptase Polymerase Chain Reaction for Severe Acute Respiratory Syndrome Coronavirus 2: Role of Deep-Learning-Based CT Diagnosis and Insights from Two Cases. Korean J Radiol 2020; 21: 505-508.

28. Zias N, Chroneou A, Tabba MK et al. Post tracheostomy and post intubation tracheal stenosis: report of 31 cases and review of the literature. BMC Pulm Med 2008; 8: 18.

29. Stauffer JL, Olson DE, Petty TL. Complications and consequences of endotracheal intubation and tracheotomy. A prospective study of 150 critically ill adult patients. Am J Med 1981; 70: 65-76.

30. Nouraei SA, Ma E, Patel A et al. Estimating the population incidence of adult post-intubation laryngotracheal stenosis. Clin Otolaryngol 2007; 32: 411-412.

31. Nesek-Adam V, Mrsic V, Oberhofer D et al. Post-intubation long-segment tracheal stenosis of the posterior wall: a case report and review of the literature. J Anesth 2010; 24: 621-625.

32. Storm B, Dybwik K, Nielsen EW. Late complications after percutaneous tracheostomy and oral intubation: Evaluation of 1,628 procedures. Laryngoscope 2016; 126: 1077-1082.

33. Yu F, Yan L, Wang N et al. Quantitative Detection and Viral Load Analysis of SARS-CoV-2 in Infected Patients. Clin Infect Dis 2020.

34. Ramanathan K, Antognini D, Combes A et al. Planning and provision of ECMO services for severe ARDS during the COVID-19 pandemic and other outbreaks of emerging infectious diseases. Lancet Respir Med 2020.

35. Henry BM. COVID-19, ECMO, and lymphopenia: a word of caution. Lancet Respir Med 2020.

36. MacLaren G, Fisher D, Brodie D. Preparing for the Most Critically Ill Patients With COVID-19: The Potential Role of Extracorporeal Membrane Oxygenation. JAMA 2020.

37. Shen C, Wang Z, Zhao F et al. Treatment of 5 Critically Ill Patients With COVID-19 With Convalescent Plasma. JAMA 2020.

38. Wu J, Li W, Shi X et al. Early antiviral treatment contributes to alleviate the severity and improve the prognosis of patients with novel coronavirus disease (COVID-19). J Intern Med 2020.

39. Brass P, Hellmich M, Ladra A et al. Percutaneous techniques versus surgical techniques for tracheostomy. Cochrane Database Syst Rev 2016; 7: CD008045. 
40. Boran OF, Bilal B, Bilal N et al. Comparison of the efficacy of surgical tracheostomy and percutaneous dilatational tracheostomy with flexible lightwand and ultrasonography in geriatric intensive care patients. Geriatr Gerontol Int 2020; 20: 201-205.

41. Iftikhar IH, Teng S, Schimmel M et al. A Network Comparative Meta-analysis of Percutaneous Dilatational Tracheostomies Using Anatomic Landmarks, Bronchoscopic, and Ultrasound Guidance Versus Open Surgical Tracheostomy. Lung 2019; 197: 267-275.

42. Flum DR, Steinberg SD, Adams PX, Wallack MK. Bedside percutaneous tracheostomy in acquired immunodeficiency syndrome. Am Surg 1998; 64: 444-446.

43. Ahmed N, Hare GM, Merkley J et al. Open tracheostomy in a suspect severe acute respiratory syndrome (SARS) patient: brief technical communication. Can J Surg 2005; 48: 68-71.

44. Gobatto AL, Besen BA, Tierno PF et al. Comparison between ultrasound- and bronchoscopy-guided percutaneous dilational tracheostomy in critically ill patients: a retrospective cohort study. J Crit Care 2015; 30: 220 e213-227.

45. Song J, Xuan L, Wu W et al. Comparison of Percutaneous Dilatational Tracheostomy Guided by Ultrasound and Bronchoscopy in Critically Ill Obese Patients. J Ultrasound Med 2018; 37: 1061-1069.

46. Gobatto ALN, Besen B, Tierno P et al. Ultrasound-guided percutaneous dilational tracheostomy versus bronchoscopy-guided percutaneous dilational tracheostomy in critically ill patients (TRACHUS): a randomized noninferiority controlled trial. Intensive Care Med 2016; 42: 342-351.

47. Kwan A, Fok WG, Law KI, Lam SH. Tracheostomy in a patient with severe acute respiratory syndrome. Br J Anaesth 2004; 92: 280-282.

48. Wei WI, Tuen HH, Ng RW, Lam LK. Safe tracheostomy for patients with severe acute respiratory syndrome. Laryngoscope 2003; 113: 1777-1779.

49. Zamora JE, Murdoch J, Simchison B, Day AG. Contamination: a comparison of 2 personal protective systems. CMAJ 2006; 175: 249-254.

50. Tompkins BM, Kerchberger JP. Special article: personal protective equipment for care of pandemic influenza patients: a training workshop for the powered air purifying respirator. Anesth Analg 2010; 111: 933-945.

51. Prompetchara E, Ketloy C, Palaga T. Immune responses in COVID-19 and potential vaccines: Lessons learned from SARS and MERS epidemic. Asian Pac J Allergy Immunol 2020; 38: 1-9.

52. Xing Y, Mo P, Xiao Y et al. Post-discharge surveillance and positive virus detection in two medical staff recovered from coronavirus disease 2019 (COVID-19), China, January to February 2020. Euro Surveill $2020 ; 25$.

\section{Hosted file}

HED-20-0468_COVID_Tracheostomy_Recommendations_FINALHNFig_1.docx available at https://authorea . com/users/312328/articles/443264-tracheostomy-during-the-cov-sars-cov-2-pandemic-recommendationsfrom-the-new-york-head-and-neck-society

\section{Hosted file}

HED-20-0468_COVID_Tracheostomy_Recommendations_FINALHNFig_2.docx available at https : //authorea . com/users/312328/articles/443264-tracheostomy-during-the-cov-sars-cov-2-pandemic-recommendationsfrom-the-new-york-head-and-neck-society

\section{Hosted file}


HED-20-0468_COVID_Tracheostomy_Recommendations_FINALHNFig_3.docx available at https://authorea . com/users/312328/articles/443264-tracheostomy-during-the-cov-sars-cov-2-pandemic-recommendationsfrom-the-new-york-head-and-neck-society 